\title{
STRUCTURE AND THERMAL PROPERTIES OF MALEATED LIGNIN-RECYCLED POLYSTYRENE COMPOSITES
}

\author{
JUSTO LISPERGUER ${ }^{1,2 *}$, CHRISTIAN NUNEEZ1 PATRICIO PEREZ-GUERRERO ${ }^{2,3}$ \\ ${ }^{1}$ Departamento de Química.Facultad de Ciencias. Universidad del Bío-Bio.Collao 1202. Concepción, Chile. \\ ${ }^{2}$ Centro de Investigación de Polímeros Avanzados. CIPA. Conicyt-Regional R08C1002. Beltrán Mathieu 224. Concepción,Chile. \\ ${ }^{3}$ Departamento de Ingeniería en Maderas. Facultad de Ingeniería.Collao 1202. Concepción, Chile.
}

(Received: April 19, 2013 - Accepted: September 6, 2013)

\begin{abstract}
In this work, the changes caused by a chemical modification of the properties and chemical structures of lignin are studied. Lignin from a mixture of Eucalyptus globulus and Eucalyptus nitens obtained through the kraft pulping process was used. The lignin was isolated by acid precipitation and modified by a reaction with maleic anhydride. Maleated lignin (ML) was incorporated to recycled polystyrene (rPS) at 2, 5, and 10 wt- $\%$ by a melt-blending process. Fourier transformed infrared spectroscopy (FT-IR) was used to determine the ML structure, and the morphology of rPS/ ML composites was studied by scanning electron microscopy (SEM).

Differential scanning calorimetry (DSC) and thermogravimetric analysis (TGA) were used to study the glass-transition temperature ( $\mathrm{T}_{\mathrm{g}}$ ) and the thermal stability of rPS and rPS/ ML composites and were compared with virgin PS. The $\mathrm{T}_{\mathrm{g}}$ values of rPS and their composites were higher than those of the virgin PS. TGA revealed that the thermal stability of rPS with 2 and $5 \%$ of ML was slightly greater than that of the virgin PS. These results suggest that postconsumer PS can be used to obtain composite materials with good thermal properties.
\end{abstract}

Keywords: Maleated lignin, recycled polystyrene, thermal properties, morphology.

\section{INTRODUCTION}

Lignin is considered the second most abundant natural polymer and it is mainly obtained as a by-product in the pulp and paper industry.

This polymer is composed by phenylpropane units connected by different types of linkages, forming a branched structure containing diverse functional groups, mainly hydroxyl, methoxyl, carbonyl and carboxy, which impart a high polarity to the lignin macromolecule. ${ }^{1,2,3}$

The influence of unmodified lignin, on different types of synthetic polymers has been studied.

The effect of five different unmodified lignins and lignin derivatives on poly vinyl chloride (PVC) were studied. A major drawback of all lignins is that they diminish the impact strength of the blends in comparison with that of PVC control. In addition, the weathering stability of the blends was lower than that of PVC control.

A slight decomposition of lignins caused by the high temperature imposed by rigid PVC processing could partially explain their effect on the impact strength and weathering stability of the blends ${ }^{4}$.

Alkaline lignin isolated from sugar cane bagasse blended with polar polymers as polyvinyl alcohol (PVA), showed an increase of thermal stability due at least some miscibility of the lignin / PVA blend. The mechanical properties of the blends were not reported ${ }^{5}$.

Lignin as powder product of the prehydrolysis of beech wood was used as filler on low density polyethylene (LDPE) and polypropylene (PP). In small concentration lignin acts as a stabilizer for PP, but over $10 \%$ acts as an initiator of degradation. On LDPE, lignin acts as a stabilizer at concentrations greater than $10 \%$. For both polymers (PP and LDPE), the mechanical properties decrease with increasing lignin content ${ }^{6}$.

Alkali lignin from bamboo was used to prepare poly(4-vinylpyridine) (PVP) / lignin blend film by the casting method. The glass transition temperature $\left(\mathrm{T}_{\mathrm{g}}\right)$ of these blends increased with the increase of lignin content, which indicated that these blends were able to form a miscible phase due to the formation of intermolecular hydrogen bond between the hydroxyl of lignin and the pyridine ring of poly (4-vinyl pyridine). The thermostability of these blends decreased with the increase of lignin content. Initially, an appreciable increase in the measured tensile strength was achieved with a lignin content of $15 \%$, at which the maximum value of tensile strength was reached but, the strain behaviour of these blend films was deteriorated ${ }^{7}$.In other study, commercial lignin (Protobind 100SA-140) was used as filler with and without silica, on polysiloxane as polymeric matrix. The results of the thermomechanical, physical, and thermal investigations have evidenced the fact that lignin inserted in a silicon matrix does not alter its properties, and in some cases (e.g. mechanical properties), it even induces a slight improvement of these, leading to the conclusion that lignin can be used as an extender for silicones, with positives effects mainly on the material's cost ${ }^{8}$

The miscibility of hardwood Kraft lignin with several synthetic polymers, polyethylene oxide (PEO), polyethylene terephthalate (PET), PP and PVA, has been analysed using thermal analysis and FT-IR spectroscopy. Miscible blends were observed in lignin / PEO and lignin / PET blends, while immiscible blend behavior in the blends of lignin / PP and lignin / PVA were reported ${ }^{9}$.

Lignin from beech wood pre-hydrolysis with dioxane water mixture was used to study the oxidative stability of PP and recycled PP. Lignin was used in the concentration range from 0.5 to $10 \mathrm{wt}-\%$ with a commercial antioxidant (Irganox 1010). The antioxidant effect is increased with lignin concentration up to 5 wt- $\%{ }^{10}$.

Some semi-interpenetrating polymer networks based on vinyl ester resin and ammonium lignosulfonate modified lignin have been synthesized and characterized. The synthesized networks showed good compatibility and a slight effect of photostabilization of the resin was noticed ${ }^{11}$.

In general, all studies conclude that lignin is a promising compound to be used in polymers because of its phenolic base structure, which could lead to improvement the mechanical properties, thermal stability, photodegradation strength and UV absorber.

The influence of lignin in thermomechanical and physical properties of synthetic polymers depends on the degree of miscibility between lignin and the polymer. Recently published data indicates a low miscibility between unmodified lignin and nonpolar polyolefins such as PE, PP, PS or PVC. Miscibility increases with polymers with higher polarity as PVA, PET, PVP and PEO',12,13.

Maleic anhydride grafted polypropylene (MAPP) is a commercial coupling agent used in the manufacture of wood-polypropylene composites. MAPP slightly improves the mechanical properties of mixtures. There are several studies that seek to use other compounds with greater efficiency ${ }^{14,15,16}$.

The aim of this study is focused on chemical modification of lignin, from black liquor of Kraft pulping of Eucalyptus globulus and Eucalyptus nitens, with maleic anhydride (MA) and study its effect on processing, thermal, and morphological properties of materials based on recycled polystyrene (rPS).

The aliphatic and aromatic hydroxyl functional groups present in lignin structures provide a possibility for an esterification reaction with maleic anhydride (MA), to introduce maleate half-ester groups to the molecule. The double bond of the maleate half-esters are reactive with nonpolar olefins as PE, PP, PS or PVC.

Figure 1 shows the chemical reactions that can occur in the esterification reaction of lignin with maleic anhydride and the blend of maleated lignin with polystyrene. 


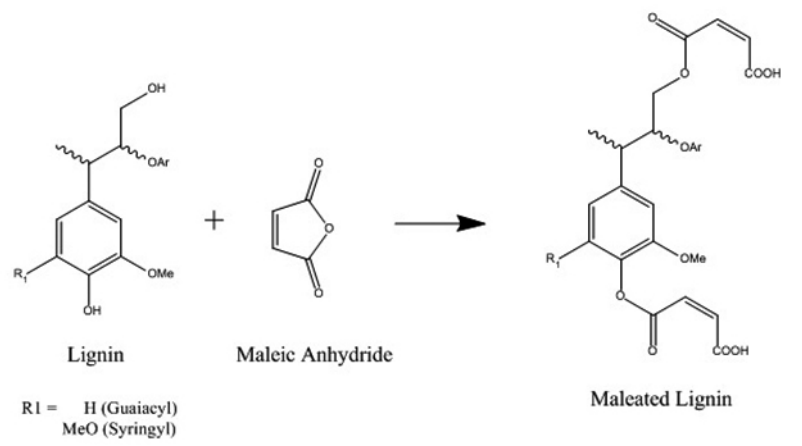

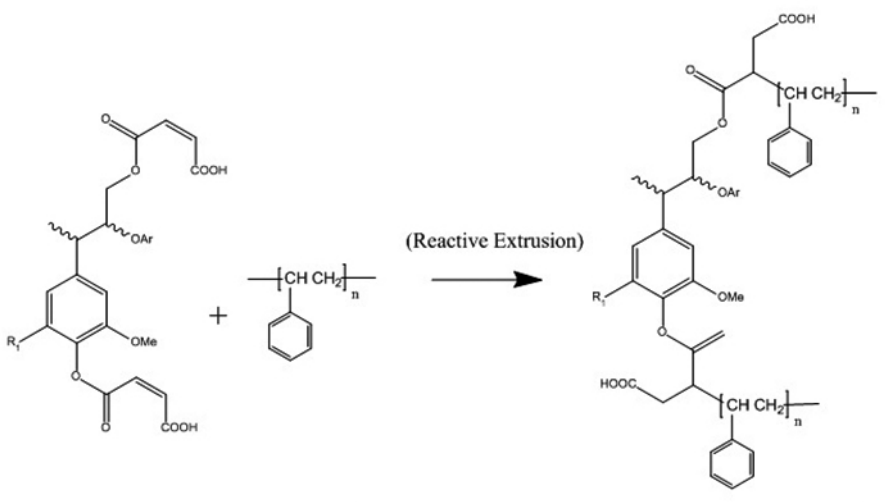

Maleated Lignin $\quad$ Polystyrene $\quad$ Polystyrene grafted with maleated Kraft lignin

Figure 1. Esterification reaction of lignin with maleic anhydride and a likely grafting reaction with polystyrene.

\section{EXPERIMENTAL}

\section{Pulping and isolation of lignin}

The lignin used in this research was obtained through a Kraft pulping of Eucalyptus globulus and Eucalyptus nitens. Four trials with 2,5 kg of chips from each species were processed separately in a $25 \mathrm{~L}$ lab digester. The digester was fed with 2,5 kg of dry wood. Sodium hydroxide $(\mathrm{NaOH})$ and sodium sulfide $\left(\mathrm{Na}_{2} \mathrm{~S}\right)$ were used as reagents for the white liquor for pulping. The white liquor and water were added at a 4:1 liquor/wood ratio. The equipment was closed and heated to reach the cooking temperature of $158^{\circ} \mathrm{C}$. The heating time was $90 \mathrm{~min}$, and the cooking time was $74 \mathrm{~min}$.

The isolation of lignin was obtained through the following method: the black liquor was concentrated in a rotary evaporator to remove most of the liquid phase. The initial $\mathrm{pH}$ of the concentrated black liquor was measured. It was precipitated with 2 molar sulfuric acid by adding the acid gradually and with constant agitation until reaching a $\mathrm{pH}$ of 3 . This solution was filtered and washed with distilled water and hydrochloric acid in turns. The resulting solid was diluted in sodium hydroxide, it was filtered again, and the liquid was precipitated with 2 -molar sulfuric acid. It was filtered and washed again with distilled water and hydrochloric acid. The solid was dried at $60^{\circ} \mathrm{C}$ for $24 \mathrm{~h}$.

\section{Esterification of lignin}

$30 \mathrm{~g}$ of maleic anhydride $\left(99 \%\right.$ purity, Merck) was heated to $100^{\circ} \mathrm{C}$ in a round-bottom flask equipped with a temperature controller. As the MA melted, $30 \mathrm{~g}$ of lignin ( $10 \mathrm{~g}$ E.nitens and $20 \mathrm{~g}$ E.globulus) with $0.3 \mathrm{~g}$ of triphenyl antimony (Fluka) as a catalyst were added. The viscous liquid reaction mixture was agitated for $5 \mathrm{~h}$ at $100^{\circ} \mathrm{C}$. The resultant product was washed with acetone at room temperature to remove unreacted MA. The maleated lignin (ML) was dried at $60^{\circ} \mathrm{C}$ for $48 \mathrm{~h}$. The sample was weighted to determine the weight percent gain. The weight percent gain by lignin from E.nitens and globulus was $12.5 \%$.

\section{Melt-blending process}

Expanded polystyrene (EPS) from packing materials from supermarkets was used in this study. The plastic waste was washed with hot water and drying to constant weight for $8 \mathrm{~h}$ at $80^{\circ} \mathrm{C}$. Afterwards, EPS was pressed at $120^{\circ} \mathrm{C}$ and 4 bar of pressure to eliminate the high air content in EPS. Afterwards, the material was pre-ground in a knife mill to an average size of 1 to $2 \mathrm{~mm}$ and then oven dried at $80^{\circ} \mathrm{C}$ for $24 \mathrm{~h}$.

Virgin polystyrene (PS STYRON 678 D, crystal) with a melt flow index value of $10 \mathrm{~g} / 10 \mathrm{~min}$ and a density of $1.05 \mathrm{~g} / \mathrm{cm}^{3}$ was supplied by the Dow Chemical Co.

Composites of ML/rPS were prepared at three content percentage of lig$\operatorname{nin}: 2,5$, and 10 weight- $\%$.

In order to evaluate the influence of lignin on the thermal and morphological properties of the rPS, virgin and recycled PS without lignin were also prepared.

The mixes were prepared in a Thermo Haake Polydrive Rheomix 600 supplied with a roller blade mixer, set at a heating temperature of $190^{\circ} \mathrm{C}$ with a rotational speed of the mixer's rollers $60 \mathrm{rpm}$, for $15 \mathrm{~min}$.

The extrudates were then molded in a laboratory press at $190^{\circ} \mathrm{C}$ and 120 bar of pressure to produces specimens for the property evaluations of the lignin-rPS composites.

Table 1 sumarizes the compositions of the polymers and lignin/rPS composites.

Table 1. Composition of the lignin/rPS composites

\begin{tabular}{|c|c|c|c|}
\hline Sample & Virgin PS (\%) & rPS (\%) & ML (\%) \\
\hline 1 & 100 & -------- & -------- \\
\hline 2 & ------------ \\
\hline 3 & ---------- & 100 & 2 \\
\hline 4 & ---------- & 98 & 5 \\
\hline 5 & ------------ & 95 & 10 \\
\hline
\end{tabular}

\section{FTIR Spectroscopy and SEM}

Lignin with and without modification was analyzed to determine the changes in the structure of the functional groups. An equipment FT-IR Perkin Elmer Spectrum Two was used to determine the chemical differences. In order to determine the morphology of the particles, SEM was used by means of a JEOL microscope (model jsm $6610 \mathrm{Iv}$ ).

\section{TGA and DSC analysis}

In order to determine the thermal behavior of the composites, a dynamic test was carried out with a TGA Q50 TA Instruments. The samples of lignin/rPS composites with an average mass of $8 \mathrm{mg}$ were heated from $50^{\circ} \mathrm{C}$ to $600^{\circ} \mathrm{C}$ at a $10^{\circ} \mathrm{C} / \mathrm{min}$ of heating rate under a nitrogen atmosphere. The thermal stability of the composites was compared with pure rPS and virgin PS.

The $T g$ of the samples was determined by the use of a DSC 822e Mettler Toledo equipped with a data analyzing software STARe. For this purpose, the ASTM 3418 standard was applied within a range of $35^{\circ} \mathrm{C}$ and $200^{\circ} \mathrm{C}$ and a heating rate of $20^{\circ} \mathrm{C} / \mathrm{min}$ under a nitrogen atmosphere.

\section{RESULTS AND DISCUSSION}

\section{FT-IR analysis of lignins}

Analyses were carried out to determine the changes generated in the structure of lignin by FT-IR. Figure 2 shows the FT-IR spectrum of lignin from a mixture of E. nitens and E. globulus with and without modification. The area between 800 and $4000 \mathrm{~cm}^{-1}$ was analyzed.

As shown in Figure 2 in the lignin without modification, it can be clearly observed that there is a presence of a wide band in $3405 \mathrm{~cm}^{-1}$, which shows the presence of the hydroxyl groups (OH stretching vibration) ${ }^{4,17,18}$. Due to the esterification, the intensity of this band is reduced, which is clearly observed in the maleated lignin.

The reduction of this band is very important since it shows the reduction in the polarity of lignin as maleate groups or carbonyl groups replace the hydroxyl groups. 


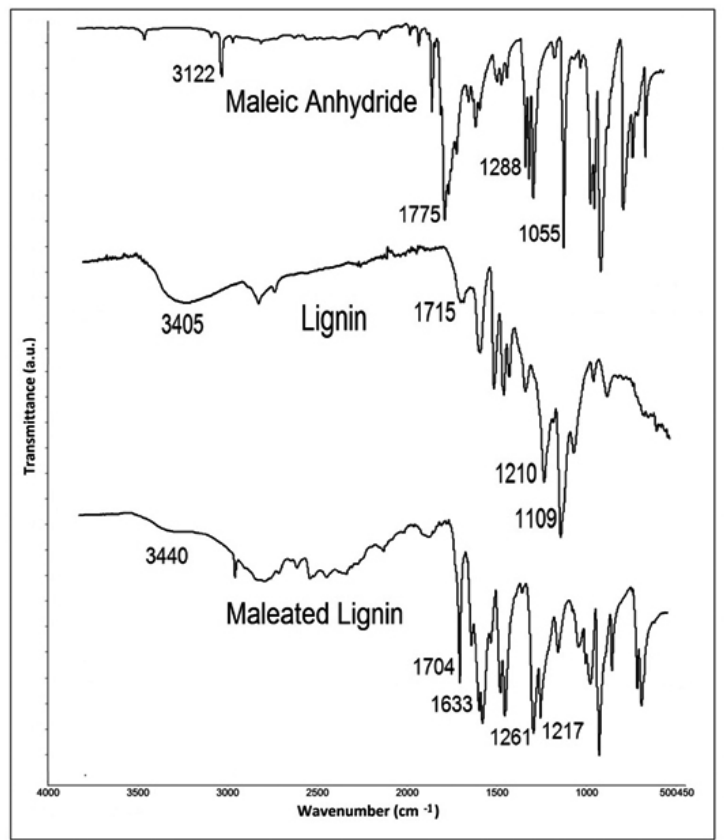

Figure 2. FTIR spectra of maleic anhydride, pure lignin, and maleated lignin.

Following, a range of signs around 2940 (C-H stretching) and $2840 \mathrm{~cm}^{-1}$ (C-H tensor) typical in lignin are observed.

Other important structures in lignin are the aromatic rings, which are present in the bands 1400 to $1600 \mathrm{~cm}^{-1}$. The most outstanding change in this area is the reduction of the intensity of the bands, as observed in Figure $2^{19,4,20,21}$

Lastly, the typical signs of the monomers, guayacil and syringyl (1210, $1109 \mathrm{~cm}^{-1}$ ), are observed with a slight intensity ${ }^{22,23}$. The chemical modification affects by slightly moving its vibrations both to the right and left.

In the maleation, the bands that indicate a chemical modification are: $2800 \mathrm{~cm}^{-1}(-\mathrm{COOH}),\left(\mathrm{C}-\mathrm{O}\right.$, ester), and the range between 1633 and $1217 \mathrm{~cm}^{-1}$ $(\mathrm{C}=\mathrm{C})$, where the band in $1633 \mathrm{~cm}^{-1}$ is the one with the highest intensity ${ }^{24,22}$. The range between 1633 and $1217 \mathrm{~cm}^{-1}$ shows that there is modification in the structure with the addition of a double bond $\mathrm{C}=\mathrm{C}$ of the maleic anhydride. The weak shoulder at about $1715 \mathrm{~cm}^{-1}$ in unmodified lignin representing $\mathrm{C}=\mathrm{O}$ stretching vibrations shows a detectable increase in intensity; it become a peak located at 1704 in the maleated lignin.

\section{Thermal properties}

Figure 3 shows the DSC thermograms of the virgin PS, rPS and the lignin/ rPS composites.

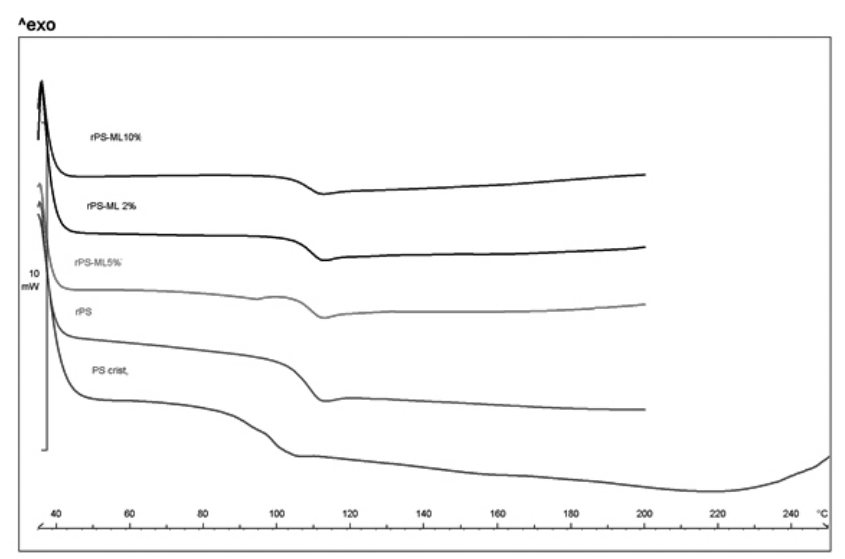

Figure 3. DSC thermograms of virgin PS, rPS and ML/rPS composites.
A comparison between the two kinds of PS showed a higher $\mathrm{T}_{\mathrm{g}}$ for rPS (ca. $12^{\circ} \mathrm{C}$ above that of the virgin PS). The lignin/rPS composites have similar $\mathrm{T}_{\mathrm{g}}$ values respect to $\mathrm{rPS}$.

The higher $\mathrm{T}_{\mathrm{g}}$ values of $\mathrm{rPS}$ and their derived composites with respect to virgin PS were probably due to the additives used in the manufacture of the EPS. These additives had some degree of physical adhesion with PS, which suggests that the chain mobility was restricted, which increased $\mathrm{T}_{\mathrm{g}}$.

Figure 4 shows the decomposition of the virgin PS, rPS and rPS/ML composites

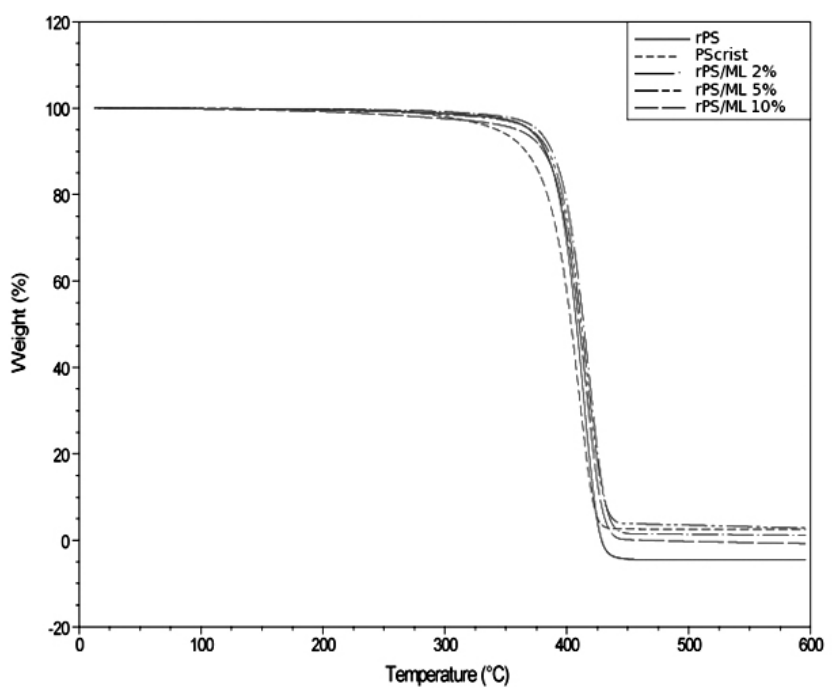

Figure 4. TGA curves of virgin PS (PS crist), rPS, rPS/ML2\%, rPS/ ML5\%, and $\mathrm{rPS} / 10 \%$.

As shown in figure 4, the decomposition profiles of the all samples occurred in one step. Virgin PS has lower thermal stability with a decomposition between 342 and $431{ }^{\circ} \mathrm{C}$. The thermal stability of rPS is slightly increased with the incorporation of 2 and $5 \%$ of maleated lignin. The incorporation of $10 \%$ of ML decreases the thermal stability of the composites.

Table 2 shows the decomposition start (at $5 \%$ decomposition), the maximum weight loss rates (derivative thermogravimetric, DTG) and the final decomposition temperature of the samples.

Table 2. TGA values of virgin PS, rPS and ML/rPS composites.

\begin{tabular}{|c|c|c|c|}
\hline Sample & $\begin{array}{c}\text { Decomposi- } \\
\text { tion(5\%) } \\
\left({ }^{\circ} \mathbf{C}\right)\end{array}$ & $\begin{array}{c}\text { DTG } \\
\left({ }^{\circ} \mathbf{C}\right)\end{array}$ & $\begin{array}{c}\text { Final temperature } \\
\left({ }^{\circ} \mathbf{C}\right)\end{array}$ \\
\hline PS & 342.7 & 409.9 & 431.3 \\
\hline rPS & 369.8 & 413.2 & 444.5 \\
\hline rPS/ML 2\% & 376.1 & 417.5 & 449.7 \\
\hline rPS/ML 5\% & 370.5 & 415.9 & 438.8 \\
\hline rPS/ML 10\% & 359.4 & 414.3 & 440.6 \\
\hline
\end{tabular}

The similar T values for rPS and rPS/ML composites and the decomposition profiles in only one step suggest some degree of miscibility between ML and the polymer. The maleation reaction increases the dispersion process of lignin in the polymeric matrix of rPS. The immiscibility of unmodified lignin in other polymers shifts the $\mathrm{T}_{\mathrm{g}}$ to higher values?

\section{Scanning electron microscopy (SEM)}

The morphology of lignin particles with and without modification was analyzed using SEM with phomicrographs at $5000 \mathrm{x}$ magnification. Figure 5 shows the morphology of virgin PS, rPS and rPS with 2, 5 and $10 \%$ maleated lignin. 

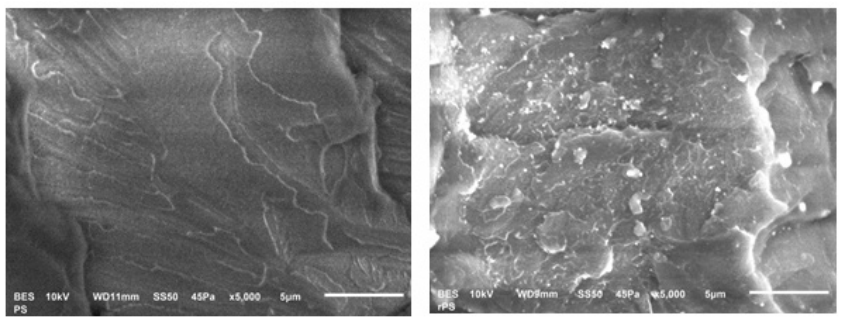

a

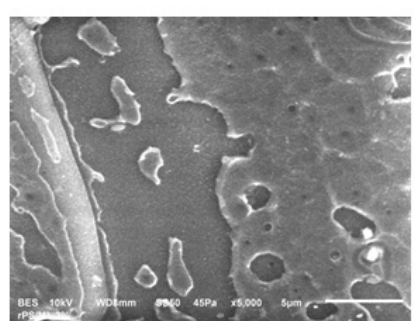

b

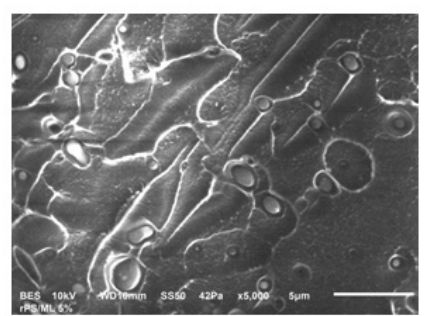

d

$\mathrm{c}$

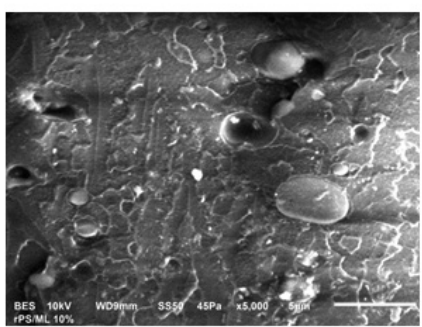

e

Figure 5. SEM images of PS, rPS and rPS/ML composites.

Figure 5 shows a clear difference in morphology between PS and rPS. The fractured surface of rPS (b) shows a greater variety of sample particles by the presence of additives incorporated into the manufacture of EPS. The virgin PS (a) shows a more homogeneous lamellar morphology. The incorporation of maleated lignin is shown in $\mathbf{c}, \mathbf{d}$ and $\mathbf{e}$ photomicrographs. Although the treated lignin appears to be in the form of particle aggregates with dimensions in the range of up to $6 \mu \mathrm{m}$, by incorporating them in the rPS matrix these aggregates break-up such that they can be found very rarely ( rPS/ML 2\%) and only in the samples where there are a higher lignin content can be appreciated.

The samples with the highest lignin content ( $\mathbf{d}$ and $\mathbf{e}$ ) appear as more homogenous in comparison with rPS and rPS/ML $2 \%$ (b and c). This suggests that although it was not expected due to the difference in polarity yet there is a good dispersal of the maleated lignin in the rPS matrix. Even more it seems that the presence of maleated lignin induces a degree of ordering in the rPS matrix (lamellar domains).

\section{CONCLUSIONS}

Lignin was isolated and purified from black liquors from the Kraft pulping of E. globulus and E. nitens. A mixture of these lignins was then esterified with maleic anhydride and blended with recycled polystyrene.

The chemical modification of the structure studied through FT-IR shows a strong reduction of the $\mathrm{OH}$ groups in maleated lignin. An increment in the band corresponding to $\mathrm{C}=\mathrm{C}$ bonds attributed to the maleic anhydride is also observed.

Other results of this study show that rPS from expanded polystyrene is a material with higher $\mathrm{T}_{\mathrm{g}}$ and thermal stability than those virgin PS.
The rPP/ML composites have similar $\mathrm{T}_{s}$ values than those $\mathrm{rPS}$. The incorporation of 2 and $5 \%$ of maleated lignin slightly increased the thermal stability of the composites. A decrease of thermal stability of rPS with $10 \%$ of lignin

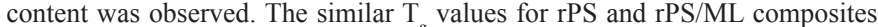
and the decomposition profiles in only one step suggest some degree of miscibility between ML and the polymer.

The SEM study shows that the samples with the highest lignin content (5 and $10 \%$ ) appear as more homogenous in comparison with rPS and rPS/ ML 2\%.

\section{ACKNOWLEDGEMENTS}

Financial support for this study was granted by the Research Center of Advanced Polymers (Centro de Investigación de Polímeros Avanzados, CIPA. Conicyt-Regional R08C1002) and DIUBB 120906 3/R project.

\section{REFERENCES}

1. J.Ralph, G. Brunow, W. Boerjan Lignins. In : Encyclopedia of life sciences, Rose, F. ; Osborne, K. (eds.) John Wiley\& Sons Ltd.,Chichester, UK, 2007.

2. K. Lundquist, J. Parkas, BioResources. 6, 920, (2011)

3. O.Faix, Hozforschung. 45, 21, (1991)

4. D.Feldman, D. Banu, J. Appl. Polym. Sci. 66, 1731, (1997)

5. E. Corradini, E.A. Gómez, A.A. Winkler, Polymer Degradation and Stability. 66, 199, (1999)

6. P. Alexy, B. Kosikova, G. Podstranska, Polymer. 41, 4901, (2000)

7. C. Liu, C. Xiao, H. Liang, J. Appl. Polym. Sci. 95, 1405, (2005)

8. G. Stiubianu, M. Cazacu, M. Cristea, A. Vlad, J. Appl. Polym. Sci. 113, 2313, (2009)

9. J.F. Kadla, S. Kubo, Composites. Part A. 35, 395, (2004)

10. A. Gregorova, Z. Cibulkova, B. Kosikova, P. Simon, Polymer Degradation and Stability. 89, 553, (2005)

11. L. Rosu, C.N. Cascaval, D. Rosu, Polymer Testing. 28, 296, (2009)

12. K. Chaochanchaikul, K. Jarayaman, V. Rosarpitak, N. Sombatsompop, Bioresources. 7, 38, (2012)

13. D.Banu, A. El-Aghoury, D.Feldman, J. Appl. Polym. Sci. 101, 2732, (2006)

14. R. Bouza, A. Lasagabaster, M.J. Abad, L. Barral, J. Appl. Polym. Sci. 109, 1197, (2008)

15. H. Zhang, W. Guo, Y. Yu, B. Li, C. Wu, Eur. Polym. J. 43,3662, (2007)

16. M. Pluta, Z. Bartczak, A. Pawlak, A. Galesky, M. Pracella, J. Appl. Polym. Sci. 82, 1423, (2001)

17. X.F. Sun, R. Sun, J.X. Sun, Journal of Agricultural and Food Chemistry 50, 6428, (2002)

18. J.Freer, J.Rodriguez, J. Baeza, N. Durán, S. Urizar,. Bol. Soc. Chil. Quim, 44, 199-2307, (1999)

19. R.J.A. Gosselink, A. Abacherli, H. Semke, R. Malherbe, P. Kauper, A. Nadif, J.E.G. van Dam, Industrial Crops and Products 19, 271, (2004)

20. M.A. Gilarranz, F. Rodríguez, M. Oliet, J. García, V. Alonso, Journal of Wood Chemistry and Technology 21, 387, (2001)

21. N. Shukry, S.M. Fadel, F.A. Agblevor, S.F. El-Kalyoubi, J. Appl. Polym. Sci. 109, 434, (2008)

22. W. Thielemans, R.P. Wool, Biomacromolecules 6, 1895, (2005)

23. C.G. Boeriu, D. Bravo, R.J.A. Gosselink, J.E.G. van Dam, Industrial Crops and Products 20, 205, (2004)

24. S. Derek, Industrial Crops and Products 27, 202, (2008) 